\title{
ANÁLISE SENSORIAL DO SUCO INTEGRAL DE UVA 'ISABEL' SUBMETIDA À APLICAÇÃO DE ÁCIDO ABSCÍSICO ${ }^{1}$
}

\author{
RENATA KOYAMA², ADRIANE MARINHO DE ASSIS ${ }^{3}$, LILIAN YUKARI YAMAMOTO², \\ SANDRA HELENA PRUDENCIO ${ }^{4}$, SÉRGIO RUFFO ROBERTO $^{5}$
}

RESUMO - O suco de uva 'Isabel' apresenta deficiência de cor e uma alternativa é o uso de ácido abscísico para aumentar a coloração. O objetivo deste trabalho foi avaliar a aceitação do suco integral de uva 'Isabel' tratada com ácido abscísico (S-ABA) em diferentes concentrações e épocas, por meio da análise sensorial, obtido em duas safras consecutivas (2011 e 2012). O delineamento experimental utilizado foi em blocos completos casualizados, com quatro repetições e cinco tratamentos: Testemunha; $S$-ABA $200 \mathrm{mg} \mathrm{L}^{-1}$ aplicado sete dias após o início da maturação (DAIM); $S$-ABA $400 \mathrm{mg} \mathrm{L}^{-1}$ aplicado aos sete DAIM; $S$-ABA $200 \mathrm{mg} \mathrm{L}^{-1}$ aplicado aos sete DAIM + $200 \mathrm{mg} \mathrm{L}^{-1}$ aplicado aos 15 dias após a primeira aplicação (DAPA); e $S$-ABA $400 \mathrm{mg} \mathrm{L}^{-1}$ aplicado aos sete DAIM $+400 \mathrm{mg} \mathrm{L}^{-1}$, aplicado aos 15 DAPA. Os sucos integrais dos tratamentos foram obtidos pelo processo 'Welch', em panela extratora por arraste de vapor. Para a avaliação sensorial, contou-se com 70 julgadores não treinados, que avaliaram os atributos: coloração, aroma, sabor, corpo e aceitação global, empregando-se uma escala hedônica de nove pontos. Verificou-se que todos os sucos integrais de uva 'Isabel' tratada com $S$-ABA apresentaram maior aceitação pelos julgadores quanto aos atributos sensoriais avaliados, sendo a concentração de $400 \mathrm{mg} \mathrm{L}^{-1}$ em uma única aplicação, aos sete DAIM, suficiente para propiciar esses resultados.

Termos para indexação: Vitis labrusca, Coloração do suco, Teste de aceitação, Antocianinas.

\section{SENSORY ANALYSIS OF 'ISABEL' GRAPE JUICE SUBMITTED TO THE APPLICATION OF ABSCISIC ACID}

\begin{abstract}
Isabel' grape juice has color deficiency and an alternative is the use of abscisic acid to increase the color. The aim of this study was to evaluate the acceptance of consumers, through sensory analysis, of 'Isabel' grape juice treated with abscisic acid in different concentrations and times; the juices were obtained in two seasons (2011 and 2012). A randomized block design was used as statistical model with four replications and five treatments: Control, S-ABA $200 \mathrm{mg} \mathrm{L}^{-1}$ applied seven days after veraison (DAV), $S$-ABA $400 \mathrm{mg} \mathrm{L}^{-1}$, seven DAV; $S$-ABA $200 \mathrm{mg} \mathrm{L}^{-1}$, seven DAV + $200 \mathrm{mg} \mathrm{L}^{-1}$, 15 days after the first application (DAFA) and $S$-ABA $400 \mathrm{mg} \mathrm{L}^{-1}$, seven DAV + $400 \mathrm{mg} \mathrm{L}^{-1}, 15$ DAFA. The juices were obtained by the 'Welch' process by steam entrainment. For juice sensory evaluation, a panel of 70 judges evaluated the following attributes: color, aroma, flavor, body and overall acceptability using a hedonic scale of nine points. It was concluded that all juices of 'Isabel' grape treated with $S$-ABA showed greater acceptance by consumers regarding all attributes evaluated, and the concentration $400 \mathrm{mg} \mathrm{L}^{-1}$ in a single application at seven DAV is enough to provide these results.
\end{abstract}

Index terms: Vitis labrusca, Juice color, Acceptance testing, Anthocyanins.

${ }^{1}$ (Trabalho 256-14). Recebido em: 23-09-2014. Aceito para publicação em : 27-05-2015

${ }^{2}$ Programa de Pós-graduação em Agronomia, Centro de Ciências Agrárias (CCA), Universidade Estadual de Londrina (UEL), Londrina, PR, Brasil. E-mail: emykoyama@hotmail.com, lilianyamamoto@yahoo.com.br

${ }^{3}$ Departamento de Agronomia, Universidade Federal de Pelotas (UFPEL), Pelotas, RS, Brasil. E-mail: agroadri@ig.com.br,

${ }^{4}$ Departamento de Ciência e Tecnologia de Alimentos, Universidade Estadual de Londrina, Londrina, PR, Brasil. E-mail: sandrah@uel.br

${ }^{5}$ Departamento de Agronomia, UEL, CP 6001, 86051-990, Londrina, PR, Brasil. E-mail: sroberto@uel.br. Autor para correspondência. 


\section{INTRODUÇÃO}

O consumo de suco de uva no Brasil tem aumentado nos últimos anos, passando de $0,15 \mathrm{~L}$ per capita em 1995 para 1,24 L em 2013. O Rio Grande do Sul é o responsável por cerca de $90 \%$ da produção nacional de suco de uvas, e sua produção em 2013 foi de 190 milhões de L de suco de uva. Nesse mesmo ano, o suco integral apresentou aumento na quantidade comercializada de $41,13 \%$ em relação ao de 2012 (MELLO, 2014).

O suco de uva pode ser classificado conforme o processo de obtenção e constituição em: integral, que apresenta concentração natural, sem qualquer adição de açúcar; concentrado, aquele parcialmente desidratado, sendo vedada a adição de açúcar; reconstituído, obtido através da diluição de suco concentrado ou desidratado até a concentração original do suco integral ou até alcançar o teor mínimo de sólidos solúveis estabelecido para suco integral; desidratado, apresenta forma sólida obtida pela desidratação do suco de uva, cujo teor de umidade não deverá ultrapassar 3\% (BRASIL, 2014).

As principais uvas utilizadas para produção de suco no Brasil são de Vitis labrusca L., dentre as quais 'Isabel', 'Concord' e 'Bordô'. A 'Isabel', uva tinta mais extensamente cultivada no País, é uma cultivar rústica e altamente fértil, proporcionando colheitas abundantes e com poucas intervenções de manejo. O suco elaborado com esta cultivar possui aroma e sabor semelhantes ao que apresenta quando consumido fresco, porém apresenta deficiência de cor devido à falta de pigmentos, havendo a necessidade de realizar cortes com sucos elaborados a partir de uvas tintureiras, como as uvas 'Concord' e 'Bordô' (SATO et al., 2008; CAMARGO et al., 2008; CAMARGO et al., 2010), as quais apresentam, entretanto, rendimentos inferiores à 'Isabel'.

A cor das bagas das uvas ocorre devido ao acúmulo de antocianinas (PEPPI et al., 2007), que começa a partir do início da maturação. $\mathrm{O}$ regulador vegetal ácido abscísico (ABA) é um dos responsáveis pela síntese e acúmulo de antocianinas nas bagas, tendo em vista que aplicações exógenas de ABA proporcionaram o aumento no teor deste flavonoide na casca de cultivares de uva de mesa (HIRATSUKA et al., 2001; BAN et al., 2003; OWEN et al., 2009). No entanto, o custo elevado para a síntese de ABA pelas companhias químicas não justificava sua utilização na agricultura (CANTÍN et al., 2007). Recentemente, foi desenvolvido um método biológico de produção de $(S)$-cis-ácido abscísico ( $S$-ABA), isômero específico da molécula de ABA, por meio do fungo Botrytis cinerea, que tornou o processo de produção para uso agrícola economicamente viável (SIEWERS et al., 2006; OWEN et al., 2009).

A partir daí, o efeito do $S$-ABA foi testado em uvas como 'Cabernet Sauvignon', 'Rubi', 'Benitaka' e 'Crimson Seedless', sendo verificado aumento na coloração das bagas (GARDIN et al., 2012; ROBERTO et al., 2012; ROBERTO et al., 2013; LEÃO et al., 2014; FERRARA et al., 2015). Koyama et al. (2014a,b) observaram que aplicações exógenas desse regulador na uva 'Isabel' aumentaram a concentração de antocianinas totais nas bagas e nos sucos. Desta forma, torna-se necessário realizar análises de aceitação dos sucos de uvas tratadas com $S$-ABA pelos consumidores.

A análise sensorial é uma ferramenta importante para mensurar a qualidade e o sucesso do suco de uva, e, desta forma, métodos científicos foram desenvolvidos para medir com precisão e reprodutibilidade as respostas humanas aos estímulos (DRAKE, 2007). Dentre os métodos existentes, a utilização do teste da escala hedônica estruturada de nove pontos tem sido amplamente usada para a coleta de dados em análises sensoriais, por ser facilmente aplicada e compreendida (REIS; MININ, 2006).

Assim, o objetivo deste trabalho foi avaliar a aceitação do suco de uvas 'Isabel' tratadas com $S$-ABA em diferentes épocas e concentrações.

\section{MATERIAL E MÉTODOS}

A aplicação do $S$-ABA foi realizada em um vinhedo comercial de uva 'Isabel' (Vitis labrusca L.) conduzida em pé-franco, com 11 anos de idade, pertencente à Vinícola Intervin ${ }^{\circledR}$, localizada em Maringá-PR $\left(23^{\circ} 25^{\prime} \mathrm{S}, 51^{\circ} 57^{\prime} \mathrm{O}\right.$, altitude de 542 $\mathrm{m})$, durante duas safras consecutivas (2011 e 2012). Segundo a classificação de Köeppen, o clima da região é do tipo $\mathrm{Cfa}$, ou seja, clima subtropical com temperatura média no mês mais frio inferior a $18^{\circ} \mathrm{C}$, e temperatura média no mês mais quente acima de $22^{\circ} \mathrm{C}$. A precipitação média anual é de $1.596 \mathrm{~mm}$, e a tendência é de concentração das chuvas nos meses de verão (IAPAR, 2010).

As videiras foram conduzidas em latada, no espaçamento de 4,0 x 1,0 m. As podas de frutificação foram realizadas de modo a deixar 2 a 3 gemas por esporão. Em seguida, aplicou-se sobre as gemas cianamida hidrogenada a $5 \%$ para induzir e uniformizar sua brotação.

O $S$-ABA foi fornecido pela empresa Valent BioScienses Corporation ${ }^{\circledR}$ (Libertyville, EUA), cuja concentração do princípio ativo é $100 \mathrm{~g} \mathrm{~L}^{-1}$.

Foram testados os seguintes tratamentos: 
a. Testemunha;

b. $S$-ABA $200 \mathrm{mg} \mathrm{L}^{-1}$ aos sete dias após o início da maturação;

c. $\quad S$-ABA $400 \mathrm{mg} \mathrm{L}^{-1}$ aos sete dias após o início da maturação;

d. $S$-ABA $200 \mathrm{mg} \mathrm{L}^{-1}$ aos sete dias após o início da maturação $+200 \mathrm{mg} \mathrm{L}^{-1}$ aos 15 dias antes da colheita;

e. $S$-ABA $400 \mathrm{mg} \mathrm{L}^{-1}$ aos sete dias após o início da maturação $+400 \mathrm{mg} \mathrm{L}^{-1}$ aos 15 dias antes da colheita.

O delineamento do experimento de campo foi em blocos completos casualizados, com cinco tratamentos e quatro repetições, sendo cada parcela composta por cinco plantas.

Para a aplicação dos tratamentos, os cachos foram pulverizados no período da manhã, utilizandose de pulverizador costal com pressão constante de 568,93 psi (39,22 bar), com bicos de pontas de jato cone oco-JA1, proporcionando cobertura completa e uniforme, sendo empregado volume de calda de 800 $\mathrm{L} \mathrm{ha}^{-1}$. Foi adicionado à calda de todos os tratamentos o espalhante não iônico Break Thru ${ }^{\circledR}\left(0,3 \mathrm{~mL} \mathrm{~L}^{-1}\right)$.

Nas duas safras avaliadas, quando o teor de sólidos solúveis (SST) das bagas atingiu ao redor de $16^{\circ}$ Brix, realizou-se a colheita manualmente.

O suco integral das uvas dos tratamentos foi elaborado pelo método "Welch", que consiste na extração do suco por arraste de vapor, utilizandose de panela extratora com capacidade de $7 \mathrm{~kg}$ (RIZZON; LINK, 2006; BORGES et al., 2013), não sendo adicionada nenhuma enzima. Foram utilizados $4 \mathrm{~kg}$ de uva de cada parcela para a elaboração do suco dos tratamentos. Para tanto, foi realizado o desengace, com a separação das bagas de sua ráquis, e, em seguida, as bagas foram lavadas em água corrente. Para o processo de extração, foi adicionada água no depósito da panela extratora, para a formação de vapor e posterior extração do suco de uva. Os sucos foram engarrafados a quente $\left(75^{\circ} \mathrm{C}\right)$ em recipientes com capacidade para $1 \mathrm{~L}$. Após o resfriamento, os sucos foram acondicionados em geladeira a $4^{\circ} \mathrm{C}$, durante seis dias.

Para a análise sensorial dos sucos de uva dos diversos tratamentos obtidos nas duas safras avaliadas, foi adotado o delineamento experimental em blocos completos casualizados, sendo realizada por 70 indivíduos não treinados, representando uma população de consumidores. As avaliações foram realizadas em cabines individuais com iluminação de luz do dia, no Laboratório de Análise Sensorial do Departamento de Ciência e Tecnologia de Alimentos da UEL. Foram avaliados os atributos: coloração, aroma, sabor, corpo (estrutura) e aceitação global das amostras de suco, usando-se uma escala hedônica de nove pontos, com extremidades denominadas: desgostei muitíssimo (1) e gostei muitíssimo (9) (MEILGAARD et al., 1999). Além disso, as notas para o atributo aceitação global foram divididas em três proporções: aprovação (\% de notas entre 6 e 9), indiferença (\% de notas 5) e rejeição (\% de notas entre 1 a 4). Para a avaliação do perfil de cada julgador do teste sensorial, foi aplicado um questionário em que foram avaliadas a faixa etária, a ocupação e a escolaridade dos entrevistados, a preferência e a frequência do consumo de suco de frutas (ANEXOS A, B e C).

As amostras foram servidas a $4^{\circ} \mathrm{C}$, em taças de acrílico transparente, na quantidade de $40 \mathrm{~mL}$ de cada amostra. Cada julgador recebeu, em ordem aleatorizada, as cinco amostras codificadas com números aleatórios de três dígitos. Água potável em temperatura ambiente foi servida aos julgadores para a limpeza da boca antes e entre as avaliações das amostras de suco de uva.

Os dados obtidos foram submetidos à análise de variância; e as médias, comparadas pelo teste de Scott-Knott, a 5\% de probabilidade.

\section{RESULTADOS E DISCUSSÃO}

Para os atributos coloração, aroma, sabor, corpo e aceitação global, os tratamentos com aplicação de $S$-ABA, independentemente da concentração e época, propiciaram as maiores médias em relação à testemunha,nas duas safras (Tabela 1). Verificou-se diferença significativa entre os tratamentos com aplicação de $S$-ABA em relação à testemunha, para todos os atributos analisados, com exceção do aroma, na safra de 2012. Quanto à coloração dos sucos, característica relacionada à concentração de antocianinas, as maiores médias foram obtidas nos tratamentos com duas aplicações de $S$-ABA, independentemente da dose, e no tratamento $S$-ABA $400 \mathrm{mg} \mathrm{L}^{-1}$, em uma única aplicação, na safra de 2011. Estes três tratamentos diferiram significativamente do tratamento $S$-ABA $200 \mathrm{mg} \mathrm{L}^{-1}$ em uma única aplicação, para a safra de 2011, que, por sua vez, diferiu significativamente da testemunha, assim como todos os tratamentos com aplicação de $S$-ABA diferiram da testemunha, na safra de 2012 .

A coloração é um componente de qualidade importante para uvas destinadas ao processamento, uma vez que, na elaboração de suco, a coloração das bagas influencia de maneira determinante o produto final (GUERRA, 2003). Em trabalho realizado por Pontes et al. (2010) com diferentes tipos de suco de 
uva, aqueles com coloração mais intensa tiveram maior aceitação dos julgadores. Camargo e Maia (2004) constataram que o suco proveniente da cultivar 'Isabel' necessita de cortes pela carência de pigmentação. Koyama et al. (2014a) observaram que a aplicação de $400 \mathrm{mg} \mathrm{L}^{-1}$ de $S$-ABA aplicados sete dias após o início da maturação resultou no aumento da concentração de antocianinas totais no suco integral da uva 'Isabel'. Estes resultados indicam que o aumento da concentração de antocianinas e, consequentemente, maior intensidade da coloração do suco integral da uva 'Isabel' alteraram a qualidade visual e refletiram em maior aceitação pelos julgadores, observada neste trabalho.

Camargo e Maia (2004) descreveram que a falta de cultivares para a realização de corte com suco de uva 'Isabel', para a melhoria da coloração, é um dos maiores entraves para o desenvolvimento de polos vitícolas emergentes em regiões subtropicais e tropicais. Entretanto, embora existam cultivares tintureiras, como BRS Rúbea, BRS Cora, BRS Violeta, BRS Carmem e BRS Magna (CAMARGO et al., 2008; EMBRAPA, 2012), deve-se considerar que estas são menos produtivas que a 'Isabel' e acabam sendo menos preferidas pelos viticultores devido ao menor lucro obtido, uma vez que as processadoras não fazem distinção de cultivares quanto ao pagamento da matéria-prima. De acordo com Borges et al. (2011), o paladar do consumidor brasileiro está habituado ao suco da uva 'Isabel', e apesar de os autores terem verificado que o corte com outras cultivares melhora seus atributos sensoriais, a utilização de uva 'Isabel' para elaboração de suco integral possui a vantagem de sua alta produtividade, disponibilidade de matéria-prima e rusticidade (CAMARGO et al., 2010).

Em relação ao aroma, sabor, corpo e aceitação global, verifica-se que a aplicação de $S$-ABA influenciou de forma positiva na análise sensorial, obtendo notas superiores à testemunha (Tabela 1). Luan et al. (2014), avaliando a influência da aplicação exógena de ácido abscísico em cachos de 'Cabernet Sauvignon', na composição do vinho, observaram maior concentração de antocianinas, fenóis totais e flavonóis em sua composição fenólica. Segundo os autores, a aplicação de ácido abscísico amplia as inter-relações entre as vias de desenvolvimento e de sinalização ambiental, que promovem ativamente a biossíntese de antocianina e também afetam a síntese de outros flavonoides, influenciando na composição e na qualidade do vinho. Portanto, possivelmente, a aplicação de $S$-ABA pode ter influenciado na composição fenólica do suco, contribuindo para sua melhor aceitação.
Nos sucos elaborados com uvas tratadas com $S$-ABA, as médias das porcentagens das respostas, para cada tratamento da aceitação global da análise sensorial, concentraram-se entre as notas 6 e 9 da escala hedônica, indicando boa aceitação dos sucos analisados pelos julgadores nas duas safras (Figura $1)$.

Quanto à avaliação do perfil dos julgadores dos sucos obtidos nas duas safras, verifica-se que a faixa etária predominante variou entre 15 e 25 anos (Figuras 2A e 3A). A maior parte dos julgadores dos sucos da safra de 2011 foi do sexo masculino, enquanto nos sucos da safra de 2012 houve equilíbrio no que se refere ao sexo dos julgadores. Quanto à ocupação e à escolaridade, predominaram principalmente estudantes e pessoas com ensino superior (Figuras 2B e 3B, e 2C e 3C).

Quanto aos tipos de suco de frutas, constatouse que, nos sucos da safra de 2011, a maioria dos julgadores do sexo masculino tem preferência por sucos de laranja e uva, enquanto os do sexo feminino preferem os sucos de maracujá, laranja e uva (Figura 2D). Na análise sensorial realizada em sucos da safra de 2012, verificou-se que, independentemente do sexo dos julgadores, a preferência dá-se por suco de laranja e uva (Figura 3D). Desta forma, observa-se que, nos sucos das duas safras avaliadas, o suco de uva foi citado por ambos os sexos, o que permite inferir que os julgadores são potenciais consumidores do suco em estudo.

Para a frequência de consumo de sucos, verifica-se que o consumo ocasional é predominante para os julgadores masculinos, enquanto para o sexo feminino variou de moderadamente e frequentemente na análise do grupo da safra de 2011 (Figura 2E) e do suco da safra de 2012, de ocasionalmente à moderadamente (Figura 3E), evidenciando que o consumo de suco é mais frequente no grupo das mulheres. Resultados semelhantes foram obtidos por Borges et al. (2011) em um experimento com suco de uva 'Isabel', em cortes com diferentes cultivares, os quais observaram que as mulheres consomem suco de frutas com maior frequência que os homens.

Em síntese, a aplicação exógena de $S$-ABA interfere de forma significativa nos atributos sensoriais do suco de uva 'Isabel', como coloração, sabor, corpo e aceitação global, sendo que $S$-ABA 400 $\mathrm{mg} \mathrm{L}{ }^{-1}$, aplicado uma vez aos sete dias após o início da maturação, é o tratamento mais indicado, pois propiciou resultados semelhantes aos tratamentos com duas aplicações de $S$-ABA nas concentrações 200 e $400 \mathrm{mg} \mathrm{L}^{-1}$, nas duas safras, representando menor custo para a aplicação do regulador vegetal. Assim, o uso de $S$-ABA em uva 'Isabel' destinada 
à elaboração de suco integral é uma alternativa promissora, pois implica o aumento da qualidade da matéria-prima, diminuindo a necessidade de cortes com sucos de outras cultivares tintureiras, visto que a coloração do suco está diretamente relacionada à aceitação do consumidor.

TABELA 1-Notas hedônicas para os atributos coloração, aroma, sabor, corpo e aceitação global de suco de uva 'Isabel' tratada com S-ABA nas safras de 2011 e 2012. Londrina-PR.

\begin{tabular}{|c|c|c|c|c|c|c|c|c|c|c|}
\hline \multirow{3}{*}{$\begin{array}{l}\text { Tratamentos } \\
\text { (concentrações em mg L-1) }\end{array}$} & \multicolumn{10}{|c|}{ Atributos } \\
\hline & \multicolumn{2}{|c|}{ Coloração } & \multicolumn{2}{|c|}{ Aroma } & \multicolumn{2}{|c|}{ Sabor } & \multicolumn{2}{|c|}{ Corpo } & \multicolumn{2}{|c|}{$\begin{array}{c}\text { Aceitação } \\
\text { Global }\end{array}$} \\
\hline & $1^{\circ}$ & $2^{\circ}$ & $1^{\circ}$ & $2^{\circ}$ & $1^{\circ}$ & $2^{\circ}$ & $1^{\circ}$ & $2^{\circ}$ & $1^{\circ}$ & $2^{\circ}$ \\
\hline Testemunha & $6,5 \mathrm{c}$ & $6,5 \mathrm{~b}$ & $5,9 \mathrm{~b}$ & $6,5 \mathrm{a}$ & $4,9 \mathrm{~b}$ & $5,5 \mathrm{~b}$ & $6,0 \mathrm{~b}$ & $6,0 \mathrm{~b}$ & $5,7 \mathrm{~b}$ & $6,1 \mathrm{~b}$ \\
\hline S-ABA 200 (7DAIM) & $7,1 \mathrm{~b}$ & $7,7 \mathrm{a}$ & $6,8 \mathrm{a}$ & $6,9 \mathrm{a}$ & $6,3 \mathrm{a}$ & $6,3 \mathrm{a}$ & $6,7 \mathrm{a}$ & $6,7 \mathrm{a}$ & $6,8 \mathrm{a}$ & $6,9 \mathrm{a}$ \\
\hline$S$-ABA 400 (7DAIM) & $7,6 \mathrm{a}$ & 7,9 a & $6,8 \mathrm{a}$ & $6,4 \mathrm{a}$ & $6,7 \mathrm{a}$ & $6,3 \mathrm{a}$ & $6,8 \mathrm{a}$ & $7,0 \mathrm{a}$ & 6,9 a & $6,9 \mathrm{a}$ \\
\hline $\begin{array}{l}S \text {-ABA } 200(7 \mathrm{DAIM})+200 \\
(15 \mathrm{DAPA})\end{array}$ & $7,7 \mathrm{a}$ & $7,7 \mathrm{a}$ & $6,4 \mathrm{a}$ & 6,9 a & $6,0 \mathrm{a}$ & $6,4 \mathrm{a}$ & $6,6 \mathrm{a}$ & $6,8 \mathrm{a}$ & $6,6 \mathrm{a}$ & $6,8 \mathrm{a}$ \\
\hline $\begin{array}{l}S \text {-ABA } 400(7 \mathrm{DAIM})+400 \\
(15 \mathrm{DAPA})\end{array}$ & $7,7 \mathrm{a}$ & 7,9 a & 6,9 a & $6,8 \mathrm{a}$ & $6,5 \mathrm{a}$ & $6,8 \mathrm{a}$ & $6,8 \mathrm{a}$ & $7,1 \mathrm{a}$ & $6,9 \mathrm{a}$ & $7,2 \mathrm{a}$ \\
\hline $\mathrm{F}$ & $15,61^{*}$ & $27,25^{*}$ & $7,06^{*}$ & 2,25 & $16,57^{*}$ & $7,00^{*}$ & $5,97^{*}$ & $8,59^{*}$ & $15,98 *$ & $9,53 *$ \\
\hline CV (\%) & 14,61 & 12,24 & 20,39 & 19,72 & 24,01 & 23,74 & 17,10 & 18,47 & 17,06 & 16,51 \\
\hline
\end{tabular}

Médias seguidas da mesma letra nas colunas não diferem entre si, pelo teste de $\operatorname{Scott} \operatorname{Knott}(p<0,05)$. Obs.: 7DAIM = 7 Dias Antes do Início da maturação; $15 \mathrm{DAPA}=15$ Dias Após Primeira Aplicação. Escala hedônica variando de 1: desgostei muitíssimo, a 9: gostei muitíssimo. $1^{\circ}$ : safra $2011 ; 2^{\circ}$ : safra 2012.
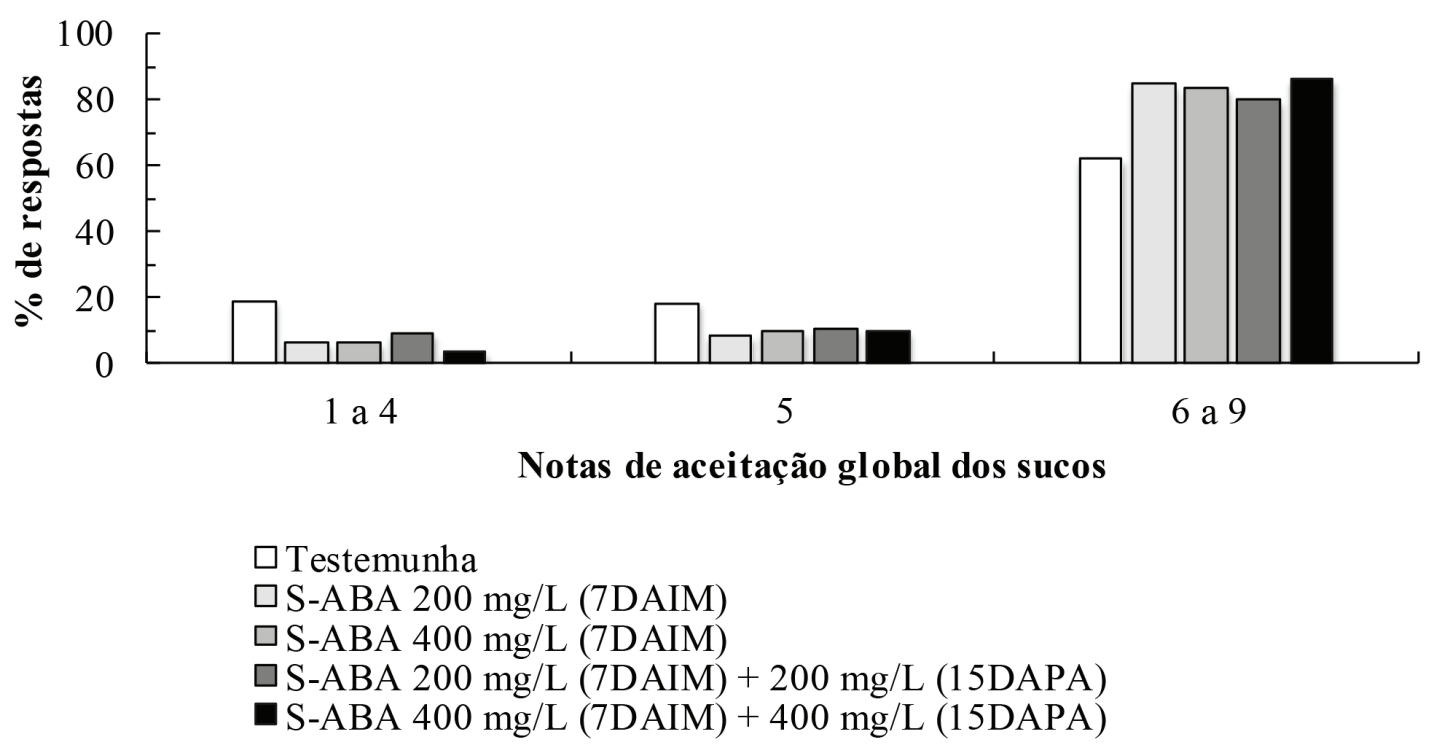

FIGURA 1- Médias das porcentagens de respostas das notas para o atributo aceitação global de suco de uva 'Isabel' tratada com $S$-ABA. Aprovação ( $\%$ de notas entre 6 e 9); indiferença ( $\%$ de notas 5 ), e rejeição (\% de notas entre 1 a 4), nas safras de 2011 e 2012. Londrina-PR. Obs.: 7DAIM = 7 Dias Antes do Início da maturação; 15DAPA = 15 Dias Após Primeira Aplicação. 


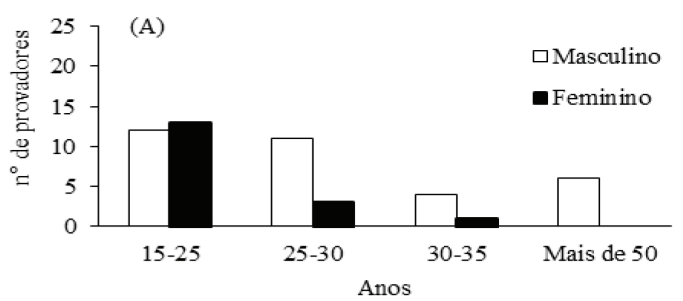

Faixa etária

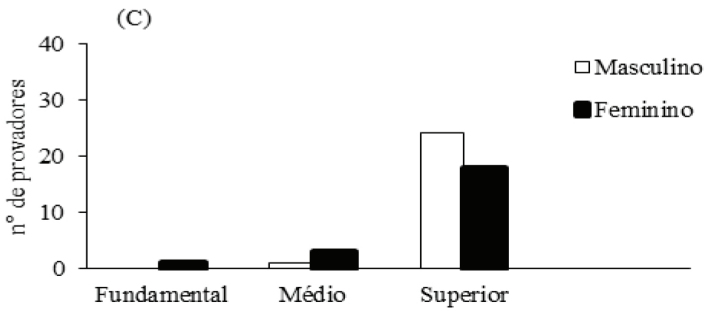

Escolaridade

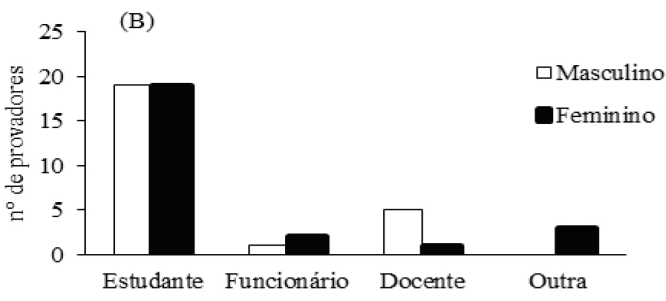

Ocupação

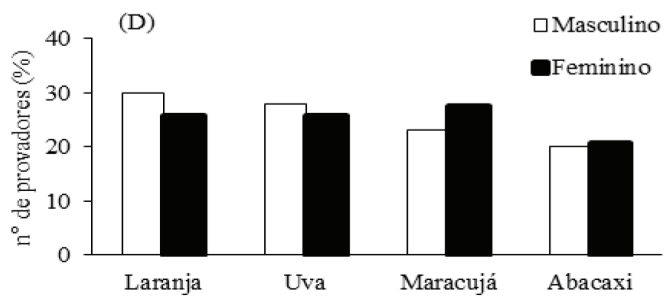

Preferência por tipo de suco

(E)

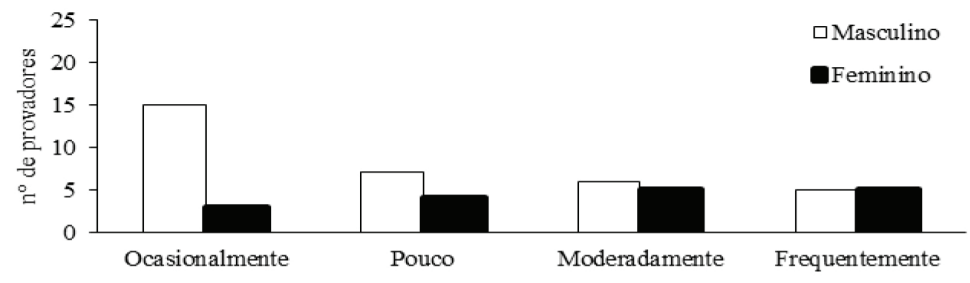

Frequência de consumo

FIGURA 2- Resultados da avaliação do perfil dos julgadores referentes à faixa etária (A); ocupação (B); escolaridade (C); preferência por tipo de suco (D), e frequência de consumo (E). Obs.: Ocasionalmente: até uma vez por semana. Pouco: uma a duas vezes por semana. Moderadamente: duas a quatro vezes por semana. Frequentemente: mais de quatro vezes por semana. Londrina-PR, sucos integrais de uva 'Isabel' tratada com $S$-ABA obtidos na safra de 2011 . 


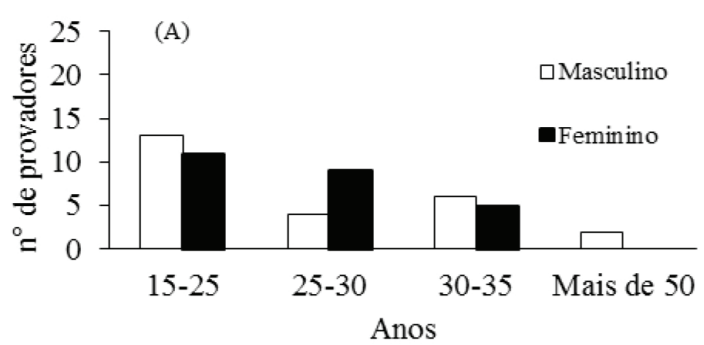

Faixa etária

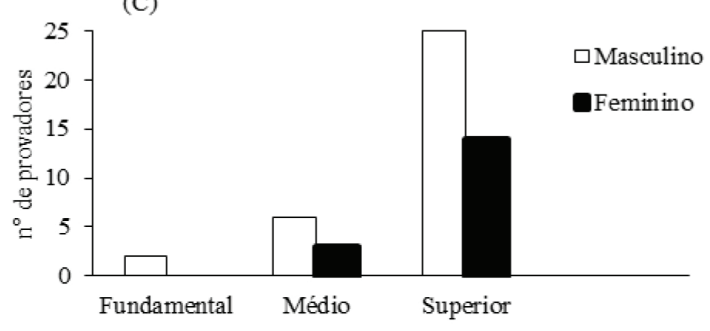

Escolaridade

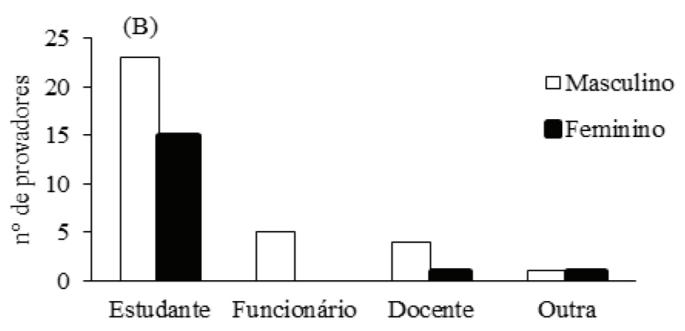

Ocupação

(D)

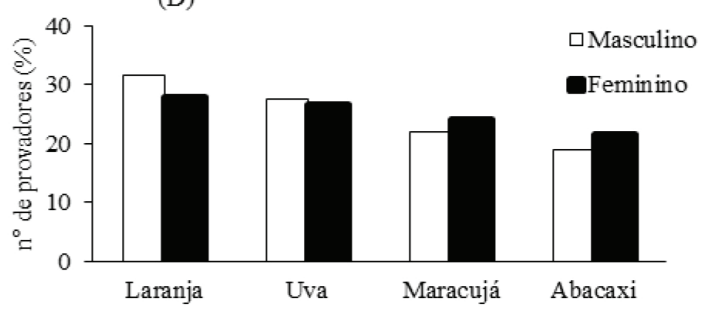

Preferência por tipo de suco

(E)

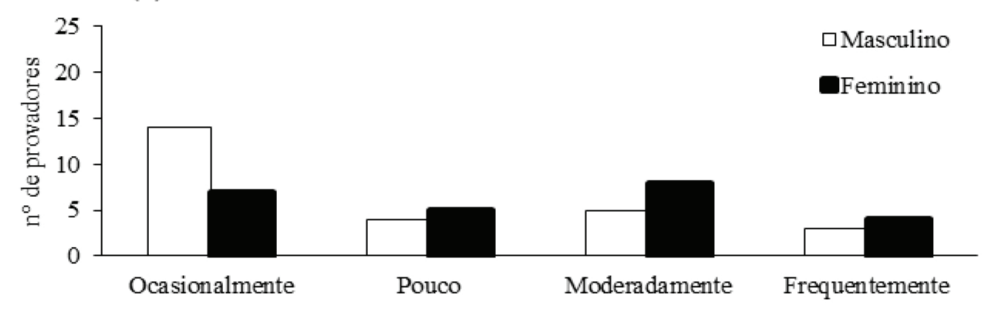

Frequência de consumo

FIGURA 3- Resultados da avaliação do perfil dos julgadores referentes à faixa etária (A); ocupação (B); escolaridade (C); preferência por tipo de suco (D), e frequência de consumo (E). Obs.: Ocasionalmente: até uma vez por semana. Pouco: uma a duas vezes por semana. Moderadamente: duas a quatro vezes por semana. Frequentemente: mais de quatro vezes por semana. Londrina-PR, sucos integrais de uva 'Isabel' tratada com $S$-ABA obtidos na safra de 2012 . 


\section{CONCLUSÃO}

O suco integral obtido de uvas 'Isabel' tratadas com $S$-ABA apresenta maior aceitação pelos julgadores quanto aos atributos sensoriais: coloração, aroma, sabor, corpo e aceitação global, sendo a concentração de $400 \mathrm{mg} \mathrm{L}^{-1} \mathrm{em}$ uma única aplicação, aos sete dias após o início da maturação, suficiente para propiciar esses resultados.

\section{REFERÊNCIAS}

BAN, T.; KOBAYASHI, S.; GOTOYAMAMOTOS, N.; HORIUCHI, S. Abscisic acid and 2, 4-dichlorohenoxyacetic acid affect the expression of anthocyanin biosynthetic pathway genes in 'Kyoho' grape berries. Journal of Horticultural Science and Biotechnology, Kent, v. 78, n. 4, p. 586-589, 2003.

BORGES, R. S.; PRUDENCIO, S. H.; ROBERTO, S. R.; ASSIS, A. M. de. Avaliação sensorial de suco de uva cv. Isabel em cortes com diferentes cultivares. Revista Brasileira de Fruticultura, Jaboticabal, v. 33, n. 1, p. 584-591, 2011.

BORGES, R. S.; SILVA, G. A.; ROBERTO, S. R.; ASSIS, A. M. de; YAMAMOTO, L. Y. Phenolic compounds, favorable oxi-redox activity and juice color of 'Concord grapevine clones. Scientia Horticulturae, Amsterdam, v. 161, p. 188-192, 2013.

BRASIL. Decreto ${ }^{\circ} 8.198$, de 20 de fevereiro de 2014. Regulamenta a Lei $n^{\circ} 7.678$, de 8 de novembro de 1988, que dispõe sobre a produção, circulação e comercialização do vinho e derivados da uva e do vinho. Diário Oficial da República Federativa do Brasil, Brasília, DF: Presidência da República, Casa Civil, 2015.

CAMARGO, U. A.; MAIA, J. D. G. BRS CORA: nova cultivar de uva para suco, adaptada a climas tropicais. Bento Gonçalves: Embrapa Uva e Vinho, 2004. 7 p.

CAMARGO, U. A.; MAIA, J. D. G.; RITSCHEL, P. S. BRS Carmem: nova cultivar de uva tardia para suco. Bento Gonçalves: Embrapa Uva e Vinho, 2008. 4 p.
CAMARGO, U. A.; MAIA, J. D. G.; RITSCHEL, P. S. Novas cultivares brasileiras de uva. Bento Gonçalves: Embrapa Uva e Vinho, 2010. 64 p.

CANTÍN, C. M. A.; FIDELIBUS, B. M. W.; CRISOSTO, C. H. Application of abscisic acid (ABA) at veraison advanced red color development and maintained postharvest quality of 'Crimson Seedless' grapes. Postharvest Biology and Technology, Amsterdam, v. 46, p. 237-241, 2007.

DRAKE, M. A. Invited Review: Sensory Analysis of Dairy Foods. Journal Dairy Science, Champaign, v. 90, n. 11, p. 4925-4937, 2007.

EMBRAPA - Empresa Brasileira de Pesquisa Agropecuária. Cultivares lançadas. 2012. Disponível em: <http://www.cnpuv.embrapa.br/ pesquisa/pmu/cultivares.html > . Acesso em: 4 jan. 2014.

FERRARA, G.; MAZZEO, A.; MATARRESE, A. M. S.; PACUCCI, C.; PUNZI, R.; FACCIA, M.; TRANI A.; GAMBACORTA, G. Application of abscisic acid (S-ABA) and sucrose to improve colour, anthocyanin content and antioxidant activity of cv. Crimson Seedless grape berries. Australian Journal of Grape and Wine Research, v. 21, n. 1, p.18-29, 2015.

GARDIN, J.P. P.; SCHUMACHER, R. L.; BETTONI, J. C.; PETRI, J. L.; SOUZA, E. L. Ácido abscísico e Etefom: influência sobre a maturação e qualidade das uvas Cabernet Sauvignon. Revista Brasileira de Fruticultura, v. 34, n. 2, p. 321-327, 2012.

GUERRA, C. C. Uva para processamento: pós-colheita. Brasília: Embrapa Informação Tecnológica: Bento Gonçalves: Embrapa Uva e Vinho, 2003. 67 p.

HIRATSUKA, S.; ONODERA, H.; KAWAI, Y.; KUBO, T.; ITOH, H.; WADA, R. ABA and sugar effects on anthocyanin formation in grape berry cultured in vitro. Scientia Horticulturae, Amsterdam, v. 90, n. 2, p. 121-130, 2001.

IAPAR - Instituto Agronômico Do Paraná. Cartas climáticas. Versão eletrônica. 2010. Disponível em: $\leq$ http://www.iapar.br/modules/conteudo/conteudo. php?conteudo=677> Acesso em: 20 dez. 2013. 
KOYAMA, R. ASSIS, A. M. D.; YAMAMOTO, L. Y.; BORGES, W. F. S.; PRUDENCIO, S. H.; ROBERTO, S. R. Exogenous abscisic acid increases the anthocyanin concentration of berry and juice from 'Isabel' grapes (Vitis labrusca L.). Hortscience, Alexandria, v.49, n.4, p.460-464, 2014a.

KOYAMA, R. YAMAMOTO, L. Y.; BORGES, W. F. S.; BONINI, M. P.; BORGES, R. S., ASSIS, A. M. D.; ROBERTO, S. R. Épocas de aplicação e concentrações de ácido abscísico no incremento da cor da uva 'Isabel'. Semina, Londrina, v.35, n. 4, p.1697-1706, 2014b.

LEÃO, P. C. S.; LIMA, M. A. C.; COSTA, J. P. D.; TRINDADE, D. C. G. Abscisic acid and ethephon for improving red color and quality of Crimson seedless grapes grown in a tropical region. American Journal of Enology and Viticulture, 2014. doi: 10.5344/ ajev.2014.14041.

LUAN, L. Y.; ZHANG, Z. W.; XI, Z. M.; HUO, S. S.; MA, L. N. Comparing the effects of exogenous abscisic acid on the phenolic composition of Yan 73 and Cabernet Sauvignon (Vitis vinifera L.) wines. European Food Research and Technology, Berlin, 2014. DOI 10.1007/s00217-014-2206-z.

MEILGAARD, M.; CIVILLE, G.V.; CARR, B.T. Sensory evaluation techniques. $3^{\text {rd }}$ ed. Boca Raton: CRC, 1999. 387p.

MELLO, L. M. R. Vitivinicultura brasileira: Panorama 2013. Bento Gonçalves: EMBRAPA, 2014. 6p. (Comunicado técnico, 156).

OWEN, S. J.; LAFOND, M. D.; BOWEN, P.; BOGDANOFF, C.; USHER, K.; ABRAMS, S. R. Profiles of abscisic acid and its catabolites in developing Merlot grape (Vitis vinifera) berries. American Journal of Enology and Viticulture, Davis, v. 60, n. 3, p. 277-284, 2009.

PEPPI, M. C.; FIDELIBUS M. W.; DOKOOZLIAN $\mathrm{N}$. Application timing and concentration of abscisic acid affect the quality of 'Redglobe' grapes. Journal of Horticultural Science \& Biotechnology, Ashford, v. 82, n. 2, p. 304-310, 2007.
PONTES, P. R. B.; SANTIAGO, S. S.; SZABO, T. N.; TOLEDO, L. P.; GOLLUCKE, A. P. B. Atributos sensoriais e aceitação de sucos de uva comerciais. Ciência e Tecnologia de Alimentos, Campinas, v. 30, n. 2, p. 313-318, 2010.

REIS, R. C.; MININ, V. P. R. Análise sensorial: estudos com consumidores. Viçosa: Ed. UFV. 2006. $83 \mathrm{p}$.

RIZZON, L. A.; LINK, M. Composição do suco de uva caseiro de diferentes cultivares. Ciência Rural, Santa Maria, v. 36, n. 2, p. 689-692, 2006.

ROBERTO, S. R.; ASSIS, A. M.; YAMAMOTO, L. Y.; MIOTTO, L. C. V.; SATO, A. J.; KOYAMA, R.; GENTA, W. Application timing and concentration of abscisic acid improve color of 'Benitaka' table grape. Scientia Horticulturae, Amsterdam, v. 142, p. 44-48, 2012.

ROBERTO, S. R.; ASSIS, A. M.; YAMAMOTO, L. Y.; MIOTTO, L. C. V.; KOYAMA, R.; SATO, A. J.; BORGES, R. S. Ethephon use and application timing of abscisic acid for improving color of 'Rubi' table grape. Pesquisa Agropecuária Brasileira, Brasília, v. 48, n. 7, p. 797-800, 2013.

SATO, A. J.; JUBILEU, B. S.; SANTOS, C. E.; BERTOLUCCI, R.; SILVA, R. A. L.; CARIELO, M.; GUIRAUD, M. C.; FONSECA, I. C. B.; ROBERTO, S. R. Fenologia e demanda térmica das videiras Isabel e Rubea sobre diferentes porta-enxertos na região norte do Paraná. Semina: Ciências Agrárias, Londrina, v. 29, n. 2, p. 283-292, 2008.

SIEWERS, V.; KOKKELINK, L.; SMEDSGAARD, J.; TUDZYNSKI, P. Identification of an abscisic acid gene cluster in the grey mould Botrytis cinerea. Applied and Environmental Microbiology, Baltimore, v. 72, n. 7, p. 4619-4626, 2006. 